\title{
Application of an adapted PRECIS-2 instrument to assess efficacy- and effectiveness-study designs in a systematic review of intervention studies of the hepatitis $C$ virus-care continuum among people who use drugs
}

This article was published in the following Dove Press journal:

Comparative Effectiveness Research

\author{
Ashly E Jordan ${ }^{1,2}$ \\ David C Perlman ${ }^{2,3}$ \\ Daniel J Smith' \\ Holly Hagan ${ }^{1,2}$
}

'New York University, Rory Meyers College of Nursing, New York, NY, USA; ${ }^{2}$ Center for Drug Use and HIV Research, ${ }^{3}$ Icahn School of Medicine, Mount Sinai Beth Israel, New York, NY, USA
Correspondence: Ashly E Jordan Center for Drug Use and HIV Research, Rory Meyers College of Nursing, New York University, 433 First Avenue - 7th floor, New York, NY 10010, USA Tel + I 2129927180 Email aj924@nyu.edu
Introduction: Systematic reviews and meta-analyses examining intervention studies may need to categorize studies by the degree to which they reflect efficacy or effectiveness study-design elements when reporting systematic reviews and meta-analysis results.

Materials and methods: We identified reports presenting data from intervention studies eligible for evaluation with an adapted PRECIS-II instrument as part of a larger systematic review of the hepatitis $\mathrm{C}$ virus (HCV)-care continuum among people who used drugs. We applied the instrument to score reports examining any of the HCV-care-continuum steps of testing, linkage to care, and treatment on an efficacy-effectiveness spectrum. Composite scores are presented in tabular format and in stacked dot plots.

Results: The adapted PRECIS-II instrument was applied to 37 unique reports that presented data on $51 \mathrm{HCV}$-care-continuum outcomes of testing $(n=16)$, linkage to care $(n=12)$, and treatment $(n=23)$. Totals of 28 , six, and three reports had been produced on one, two, or all three outcomes, respectively. Ten and eight studies described themselves as having efficacy or effectiveness designs, respectively; 33 did not specify. PRECIS-II composite scores for reports produced on testing, linkage to care, and treatment ranged widely: 1.22-5. Composite scores for reports examining $\mathrm{HCV}$ treatment indicated study designs that tended toward effectiveness (3.35), but those examining testing (3.85) or linkage (3.8) had more effectiveness-study designs ( $P=0.003, P=0.013$, respectively).

Conclusion: Reviewed reports varied widely in their use of efficacy/effectiveness-study designs, suggesting that systematic reviews and meta-analyses need to consider heterogeneity in efficacy/effectiveness study design in analyses. Most reports tended modestly toward having effectiveness designs, and treatment studies contained the most elements of efficacy-study designs. When assessing large numbers of reports with the PRECIS-II instrument, stacked dot plots may aid visually in depicting the range of scores. This review suggests that studies of the effectiveness of HCV treatment of people who use drugs at a population level is a research gap. Keywords: efficacy, effectiveness, HCV-care continuum, systematic review and meta-analysis, evidence base

\section{Plain-language summary}

Systematic reviews and meta-analyses are important methods for synthesizing existing knowledge to inform public health policy and identify research gaps. Efficacy and effectiveness studies 
provide two distinct ways to examine the impact of an intervention and address two distinct research questions, and the degree to which studies examine efficacy or effectiveness is an important form of study design that systematic reviews must address. The PRECIS-II instrument is a validated tool intended to guide intervention design and characterize degrees of efficacy and effectiveness in studies. It has been applied to trial design in various settings and in a few post hoc analyses. Using a modestly adapted PRECIS-II instrument, we sought to examine the degree to which efficacy- and effectivenessdesign elements were present in intervention studies included in a systematic review on the hepatitis $\mathrm{C}$ virus-care continuum among people who use drugs. We found that most study designs tended toward effectiveness, consistent with the recognized gap between efficacy and effectiveness in the hepatitis $C$ virus-care continuum. An important gap identified is the need for more data on the population-level effectiveness of hepatitis $\mathrm{C}$ virus treatment in broader populations of people who use drugs treated in appropriate but routine models of care.

\section{Introduction}

Systematic reviews and meta-analyses are important methods for synthesizing existing knowledge to inform public health policy and identify research gaps. ${ }^{1-3}$ There is an emerging need for systematic reviews and meta-analyses examining intervention reports to account for differences in degrees to which included reports reflect either efficacy or effectiveness designs. ${ }^{4,5}$

Systematic reviews must make assessments of the similarity of studies for inclusion on parameters, including those of study design. ${ }^{6}$ Intervention studies in particular may vary in design elements intended to maximize either internal validity, with the goal of addressing questions of the intrinsic efficacy of an intervention, or to maximize elements favoring external validity, with the goal of addressing questions of generalizability and the effectiveness of an intervention in routine practice. ${ }^{4}$ Efficacy and effectiveness studies provide two distinct ways to examine the impact of an intervention; in fact, they address two distinct research questions. ${ }^{7,8}$ Combining results from studies designed to answer different questions may lead to imprecise and possibly invalid inferences. ${ }^{9}$ The degree to which studies examine questions of efficacy or effectiveness is an important form of study-design heterogeneity that systematic reviews must address. All aspects of study design, including methods of sampling, recruiting, and data analysis, inform whether the study is asking (and answering) an efficacy- or effectiveness-research question. ${ }^{4,10}$

Hepatitis $\mathrm{C}$ virus (HCV) infection causes substantial morbidity and mortality. ${ }^{11,12}$ Morbidity and mortality persist in part due to incompletely effective and underimplemented programs to prevent primary infection and reinfection, and to very low rates of implementation of highly efficacious $\mathrm{HCV}$ treatment for those chronically infected. ${ }^{13-16} \mathrm{New}$, oral directacting antivirals with treatment regimens lasting only 8-12 weeks have more than $90 \%$ efficacy, creating the potential for HCV treatment as prevention (TasP) through lowering community HCV viral load (ie, resultant lower probabilities of $\mathrm{HCV}$ acquisition [forward transmission] among current people who inject drugs [PWID] $).{ }^{17} \mathrm{HCV}$ TasP also has the ability to reduce morbidity and mortality by curing people of their infection and thus reducing $\mathrm{HCV}$-induced hepatic injury (eg, development of cirrhosis) over the life course. However, the high cost of these medications has severely restricted access in the US, particularly for PWID, reducing the population-level effectiveness of HCV TasP. ${ }^{18}$ Modeling suggests that effective population-level HCV prevention and treatment will require broader implementation of needleexchange programs, medication-assisted treatment, and expanded treatment to serve as TasP. ${ }^{19-24}$

Published literature examining steps of the HCV-care continuum have addressed questions of both the intrinsic efficacy of antiviral agents and biobehavioral interventions, and questions of the effectiveness of these interventions in practice and at the population level. ${ }^{25,26}$ Despite the availability of HCV-testing methods with high sensitivity and specificity and treatment regimens of high efficacy, HCV has been identified as an area in which large gaps between efficacy and observed clinical and population-level effectiveness have been and in many settings continue to exist. ${ }^{26-29} \mathrm{As}$ such, systematic reviews of aspects of HCV care are likely to encounter studies reflecting various degrees of efficacy- and effectiveness-study design. ${ }^{4,30,31}$ Indeed, given the recognized efficacy-effectiveness gap, it could be argued that there is a need for the development and implementation of interventions to improve the effectiveness of $\mathrm{HCV}$-care-continuum steps.

We are conducting a systematic review and meta-analysis of the HCV-care continuum, with predefined care-continuum steps of screening or testing, links to care, treatment acceptance, initiation, and adherence, treatment completion, the biologic outcome of a sustained virologic response, and subsequent risk of reinfection. ${ }^{31}$ The intent of the systematic review was to examine data specifically for the $\mathrm{HCV}$-care continuum among people who use drugs (PWUD) as a distinct population. The rationale for the focus on this population is that PWUD as a population have distinct characteristics impacting outcomes and thus require analysis as a separate group. ${ }^{32}$ The care-continuum construct is increasingly being 
applied as a tool to examine outcomes of care for a range of conditions in which care typically consists of a series of sequential steps. ${ }^{25}$ For example, the care-continuum construct has been formally applied to public health evaluations of HIV care and to outcomes for several infectious and incommunicable diseases..$^{25,26,33,34}$ We sought to explore and characterize the degree of heterogeneity with respect to efficacy- and effectiveness-study design components among papers identified in systematic reviews examining the outcomes of HCV testing, link to care, and treatment in intervention studies using the adapted PRECIS-2 instrument ${ }^{8}$ and to develop methods to represent pooled PRECIS- 2 scores and their distribution.

\section{Materials and methods Systematic review}

The data reported here were derived from ongoing analyses being conducted as part of our systematic review/metaanalysis titled "The HCV care continuum among people who use drugs" (PROSPERO CRD42016034113). The methods of our systematic review/meta-analysis have been defined in detail. ${ }^{31}$ In brief, this protocol was developed in accordance with the Preferred Reporting Items for Systematic Review and Meta-Analysis Protocols (PRISMA-P) guidelines. ${ }^{35} \mathrm{~A}$ PRISMA flow diagram is presented in Figure 1.
The primary exposures examined in this review were PWUD or a PWID and thus at risk of HCV infection and being a former PWUD or PWID and having HCV infection. The term "PWUD" is used herein to refer to those who use or have used any illicit drug(s) by any route, but excludes those who use or have used alcohol, tobacco, or marijuana only. The term "PWID" is used to refer to those who use or have used any illicit drug by injection.

This study includes published data from both observational and experimental study designs. To be eligible, the reports needed to present outcomes of interest on any one or more of three specific HCV-care-continuum steps (testing, linkage to care, and treatment), present data from the US, be published in English between January 1, 1990 and February 20, 2016, and include data on PWUD and/or PWID. ${ }^{31}$ Inclusion criteria required that reports presented data from the US, as the progression through steps in the care continuum is highly dependent on the types of health-care delivery systems in a given country.

Initial prespecified plans were to examine and categorize reports and outcomes with respect to $\mathrm{HCV}$-care-continuum steps of screening and testing, linkage to and completion of clinical evaluations in care, interventions to increase treatment acceptance, initiation, and adherence, completion of treatment, achieving a sustained virologic response (SVR),

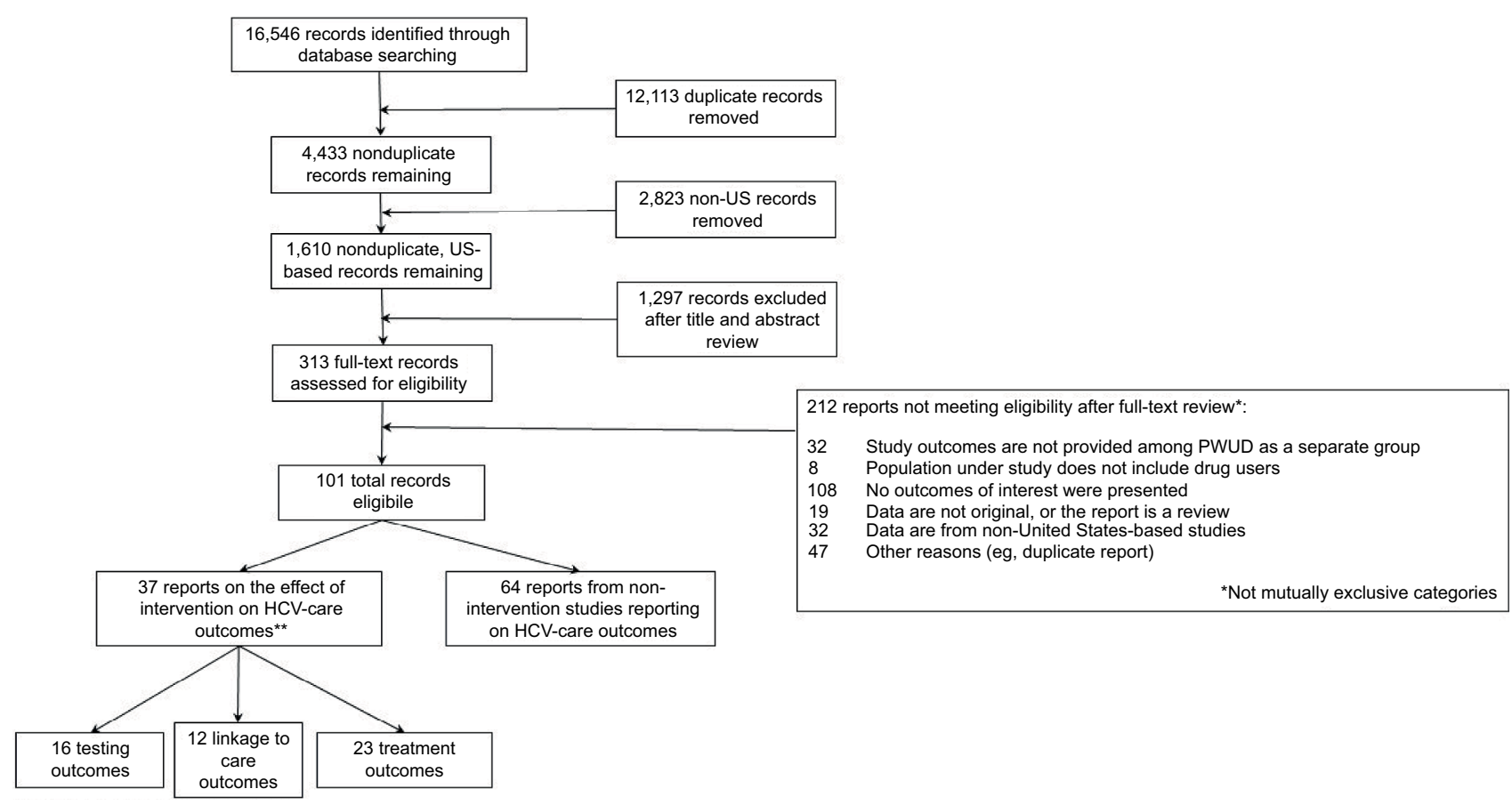

Figure I PRISMA diagram for systematic review.

Note: **Some reports reported on more than one outcome.

Abbreviations: PRISMA, Preferred Reporting Items for Systematic Reviews and Meta-Analyses; HCV, hepatitis C virus; PWUD, people who use drugs; US, United States. 
and reinfection post-SVR. Because identified reports included data on a heterogeneous mix of these steps, each defined variously, for the current analyses we recategorized these six outcomes into the following three sequential care-continuum-step outcomes: HCV screening and testing (included screening for primary infection and for recurrent infection in PWUD who may have had spontaneous clearance of initial infections), linkage to $\mathrm{HCV}$ care, including data addressing completion of HCV clinical evaluation, and HCV treatment that included treatment offers, acceptance, initiation, and completion, and outcomes of treatment (including SVR and reinfection post-SVR).

Studies were characterized by specific clinic settings, geographic location, study time period, and whether the study described itself as having an efficacy- or effectiveness-study design. Treatment studies were further categorized as to whether they examined licensed or unlicensed HCV-treatment agents. Studies were also characterized as to whether they were intervention studies (evaluating outcomes of a program or care model in either a randomized or observational design) or not. Nonintervention studies included those presenting such data as reports presenting factors associated with HCVtreatment initiation at the individual level providing no data on any implementation of a program or intervention, reports presenting prevalence data on anti-HCV and HCV RNA testing in a nonrandom selection of clinics across an area, and chart reviews of $\mathrm{HCV}$-positive patients to find reasons that they did not initiate treatment. We then applied the PRECIS-2 to identified intervention studies.

\section{Scoring on the spectrum of efficacy- effectiveness study design}

The PRECIS-2 instrument is a useful, validated tool intended to guide study design and characterize degrees of efficacy and effectiveness in studies, and it has been applied to trial design in various settings and a few post hoc analyses. ${ }^{30,36-38}$ PRECIS-2 has been shown to have good interrater reliability. ${ }^{39}$ The nine domains of PRECIS-2 are eligibility, recruitment, setting, organization, flexibility of delivery, flexibility of adherence, follow-up, outcome, and analysis.

We modestly adapted the PRECIS-2 to modify the prompts to reflect its application to the post hoc analysis of studies and to reflect considerations specifically relevant to HCV-carecontinuum studies. Using the PRECIS-2 toolkit and guided by Loudon et al, we adapted the prompts and instructions for each domain of PRECIS-2: prompts were tailored to address the post hoc nature of the review, and instructions were included that were informed by both HCV subject-matter expertise and expertise in trial design, statistical analysis, and the conduct of systematic reviews and meta-analyses. ${ }^{8,40}$ One additional consideration specific to the application of PRECIS- 2 to carecontinuum studies is that some studies present more than one outcome (eg, HCV testing and linkage to care), in which case eligibility criteria, recruitment method, and data-analysis plan may differ between outcomes. In such cases, we applied the PRECIS-2 instrument separately for each outcome for which data were presented.

Studies that presented results from intervention studies were scored with the adapted HCV PRECIS-2 instrument and were rated for each of the nine PRECIS-2 domains for each of the three possible study outcomes on which data were presented. After pilot testing, the adapted reports were reviewed by one coder (AEJ), with scores reviewed by the study team. Each domain was scored on a 5-point Likert scale from full efficacy (1) to full effectiveness (5), with a score of 3 indicating that a study-design domain was equally one of efficacy and effectiveness. In addition, a composite score is reported for each report to characterize the overall position of the design on an efficacy-effectiveness spectrum. PRECIS-2 scores for each of the included reports were depicted as wheel charts, and four of these are shown as examples (see Figures 2 and 3). Mean and median scores were calculated for each domain for all reports contributing data on each $\mathrm{HCV}$-care-continuum outcome. A series of stacked dot plots was used to represent the distribution of PRECIS-2 scores by domain and outcome (Figure 4). Statistics were calculated in SPSS and the stacked dot plots were generated in Stata. ${ }^{41,42}$

\section{Results}

The process of the systematic review is presented in Figure 1 as a PRISMA flow diagram. The initial search yielded a total of 16,546 reports. After removal of duplicate reports and studies that presented data not based in the US, 1,610 reports were assessed for eligibility by title and abstract review, 1,297 excluded for ineligibility, and the remaining 313 assessed for eligibility by full-text review. A total of 101 reports were deemed eligible for the overall systematic review; 37 reports were identified as being intervention studies specifically addressing PWUD and thus eligible for PRECIS-2 evaluation and the subject of this report.

These 37 reports presented data on 51 HCV-carecontinuum outcomes of testing $(n=16$, Table 1$),{ }^{43-58}$ linkage to care $\left(n=12\right.$, Table 2), ${ }^{44-46,49,51,52,54,57-61}$ and treatment $(n=23$, Table 3). ${ }^{44,45,51,61-80}$ A total of 28 papers reported on precisely one outcome, six papers reported on two outcomes, and three papers reported on all three outcomes. One report provided national data, four reports presented data from multiple sites, and the remaining reports were single-site studies. Reports 
presented data from 12 US states. A third of the outcomes reported data from New York. The types of settings in which the data were collected varied, but they were generally characteristic of settings where PWUD receive services (eg, needle-exchange program, methadone-maintenancetreatment program) (Tables 1-3).

Each of the nine PRECIS-2 domains was assessed for each separate outcome presented in any given report. As an example of how study design may vary with respect to efficacy-effectiveness elements in each of the PRECIS-2 domains, we describe variations identified in the domain flexibility of intervention adherence. For outcome linkage to care, an example of full efficacy was when a study required that researchers providing the intervention be highly trained and follow a strict study protocol, ${ }^{63}$ a study that used a specified protocol but that allowed for some modest protocol deviation was rated as a partial-efficacy design, ${ }^{59}$ a study that provided standard-of-care referral for off-site $\mathrm{HCV}$ care following an HCV-positive test but in which staff received some specialized training was considered to exhibit both efficacy and effectiveness elements equally, ${ }^{45}$ when a linkage to care intervention included reproducible components beyond the standard of care but that could be implemented selectively as needed, it was considered to represent a partial-effectiveness design, ${ }^{46,59}$ and a study in which linkage to care relied on offsite referral without specialized staff training or additional measures was an example of a full-effectiveness design. ${ }^{44}$

For each outcome of the HCV-care continuum, scores for each domain are presented in Tables 1-3, along with the composite score. Composite scores for reports examining $\mathrm{HCV}$ treatment were lower (3.35) than those for either testing (3.85) or linkage (3.80) $(P=0.003$ and $P=0.013$, respectively). For reports on $\mathrm{HCV}$ testing, composite scores ranged from 1.89 (SD 1.36) to 5 (SD 0). For reports on HCV linkage to care, composite scores ranged from 2.44 (SD 0.53) to 5 (SD $0)$. For reports on $\mathrm{HCV}$ treatment, composite scores ranged from 1.22 (SD 0.67) to 5 (SD 0). Reports on either testing or linkage to care tended toward effectiveness designs with means for all nine domains $>3.5$. Reports on treatment tended toward having more efficacy-design elements, particularly for the domains of analysis, delivery, and eligibility.

Most studies did not describe themselves as being efficacy or effectiveness studies (64.7\% or 33 of 51 outcomes), while ten (19.6\%) described themselves as having an efficacy design and eight (15.7\%) as having an effectiveness design. Overall composite scores for those describing themselves as an efficacy design $(n=10)$, effectiveness design $(n=8)$, and for those not specifying $(\mathrm{n}=33)$ were 3.31 (SD 1.04), 3.95
(SD 0.54), and 3.68 (SD 1.1), respectively. A total of 22 of the 23 papers reporting on $\mathrm{HCV}$ treatment among PWUD were studies of agents that were already licensed at the time of the study; one report that examined the use of an investigational treatment regimen had the lowest composite score (1.22).

Wheel charts were constructed for each of the reports (four are shown). Harris et al was one of three reports reporting on all three care-continuum steps. ${ }^{44,45,51}$ As an example, a visual depiction of adapted PRECIS-2 scores for each of the domains for each of the three outcomes reported in Harris et al is depicted in Figure 2. Figure 3 depicts the adapted PRECIS-2 scores for three of the 12 reports presenting data on linkage to care. ${ }^{46,49,58}$ A series of stacked dot plots (Figure 4) visually depict the range and distribution of all of the individual PRECIS-2 scores for each of the domains by outcome. Mean and median scores for each of the nine domains for each of the three outcomes (testing, linkage to care, and treatment) and for composite score for each reported outcome are shown in Table 4.

\section{Discussion}

There have been various approaches proposed to assist scientists in formulating their study designs to address questions of efficacy or of effectiveness, and similarly in categorizing published studies as reflecting efficacy- or effectivenessstudy designs. ${ }^{8,81,83}$ Gartlehner et al developed an instrument for distinguishing between efficacy and effectiveness studies in systematic reviews. ${ }^{82}$ This instrument dichotomized studies as being either an efficacy or effectiveness design. While the instrument was shown to have high specificity for identifying effectiveness studies, we sought an instrument that would allow us to categorize studies included in our systematic review on a spectrum of efficacy-effectiveness. ${ }^{81} \mathrm{We}$ anticipated that studies addressing the $\mathrm{HCV}$-care continuum would be likely to address questions of both the efficacy and effectiveness of diagnosis, linkage to care, and treatment efforts, as well as the impact of behavioral and structural interventions to improve the outcomes of each of these HCVcare-continuum steps. Categorizing studies along a spectrum of efficacy-effectiveness would be more meaningful and precise than relying on a dichotomous measure.

Loudon et $\mathrm{al}^{8}$ published a useful tool, the PRECIS-2, to assist scientists in designing and carrying out studies that are consistent with their research questions. Although primarily intended to assist in study design, PRECIS- 2 has also been applied post hoc to assess for the degree of efficacy or effectiveness in published trials. ${ }^{8,37}$ We have modestly adapted the PRECIS-2 instrument to allow post hoc assessments of where 
Table I PRECIS-2 assessments for testing outcome $(n=16)$

\begin{tabular}{|c|c|c|c|c|}
\hline $\begin{array}{l}\text { First author, } \\
\text { publication year }\end{array}$ & Recruitment site & Location & $\begin{array}{l}\text { Stated study } \\
\text { design }\end{array}$ & Time period \\
\hline D'Souza, $2003^{43}$ & STD $^{\text {a }}$ clinic & Houston, TX & Effectiveness & Feburary I - May 2I, $200 \mathrm{I}$ \\
\hline Gunn, $2005^{44}$ & $\begin{array}{l}\text { Nonresidential alternative } \\
\text { sentencing drug } \\
\text { rehabilitation }\end{array}$ & San Diego, CA & $\mathrm{n} / \mathrm{s}^{\mathrm{b}}$ & April 1999 - December 2002 \\
\hline Harris, $2010^{45}$ & MMTPc & New York, NY & $\mathrm{n} / \mathrm{s}$ & July 2003 - July 2005 \\
\hline Hennessy, $2007^{46}$ & STD clinic & New York, NY & $\mathrm{n} / \mathrm{s}$ & May 2000 - March 2004 \\
\hline Kapadia, $2007^{47}$ & Community & $\begin{array}{l}\text { New York, NY; Baltimore, MD; } \\
\text { Seattle,WA }\end{array}$ & Efficacy & April 2002 - May 2004 \\
\hline Kim, $2013^{48}$ & Correctional facility & Concord and Framingham, MA & $\mathrm{n} / \mathrm{s}$ & October 2006 - March 2008 \\
\hline Lally, $2005^{49}$ & $\begin{array}{l}\text { Short-term drug treatment } \\
\text { program }\end{array}$ & Fallriver, MA & $\mathrm{n} / \mathrm{s}$ & January 200I - March 200I \\
\hline Mallette, $2008^{50}$ & $V^{d}$ & Providence, RI & Effectiveness & October 1998 - March 2004 \\
\hline Martinez, $2012^{51}$ & MMTP & New York, NY & Effectiveness & July 2006 - June 2008 \\
\hline Masson, $2013^{52}$ & MMTP & New York, NY & Efficacy & $2008-2011$ \\
\hline McGarry, $2002^{53}$ & NEPe, MMTP & $\mathrm{RI}$ & $\mathrm{n} / \mathrm{s}$ & July 1997 - March 1998 \\
\hline McGovern, $2006^{54}$ & Correctional facility & MA & $\mathrm{n} / \mathrm{s}$ & November 200I - May 2004 \\
\hline Merchant, $2014^{55}$ & $\mathrm{ED}^{\mathrm{f}}$ & Providence, RI & $\mathrm{n} / \mathrm{s}$ & Feburuary 2011 - March 2012 \\
\hline Merchant, $2015^{56}$ & ED & New England & $\mathrm{n} / \mathrm{s}$ & July 2010 - December 2012 \\
\hline Morano, $2014^{57}$ & Mobile medical clinic & New Haven, CT & $\mathrm{n} / \mathrm{s}$ & $2012-2013$ \\
\hline White, $2016^{58}$ & ED & Oakland, CA & $\mathrm{n} / \mathrm{s}$ & April 2014 - October 2014 \\
\hline
\end{tabular}

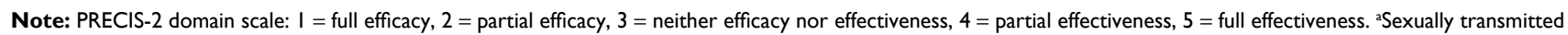
disease; ${ }^{b}$ Not specified; ' methadone maintenance treatment program; ${ }^{d}$ Veterans Affairs; ${ }^{\text {eneedle exchange program; ' } e m e r g e n c y ~ d e p a r t m e n t . ~}$ Abbreviations: STD, sexually transmitted disease; n/s, not specified; MMTP, methadone-maintenance-treatment program; VA, Veterans Affairs; NEP, needle-exchange program; ED, emergency department.

Table 2 PRECIS-2 assessments for linkage to care outcome $(n=12)$

\begin{tabular}{|c|c|c|c|c|}
\hline $\begin{array}{l}\text { First author, } \\
\text { publication year }\end{array}$ & Recruitment site & Location & Stated study design & Time period \\
\hline Brady, $2007^{59}$ & $\begin{array}{l}V^{a} \text { gastroenterology and liver } \\
\text { clinics }\end{array}$ & Durham, NC & Effectiveness & January 2002 - November 2004 \\
\hline Fishbein, $2004^{60}$ & MMTPb & New York, NY & Not specified (n/s) & November $1999-\mathrm{n} / \mathrm{s}$ \\
\hline Gunn, $2005^{44}$ & $\begin{array}{l}\text { Nonresidential alternative } \\
\text { sentencing } \\
\text { drug rehabilitation }\end{array}$ & San Diego, CA & $\mathrm{n} / \mathrm{s}$ & April 1999 - December 2002 \\
\hline Harris, $2010^{45}$ & MMTP & New York, NY & $\mathrm{n} / \mathrm{s}$ & July 2003 - July 2005 \\
\hline Hennessy, $2007^{46}$ & STD ${ }^{c}$ clinic & New York, NY & $\mathrm{n} / \mathrm{s}$ & May 2000 - March 2004 \\
\hline Lally, $2005^{49}$ & $\begin{array}{l}\text { Short-term drug treatment } \\
\text { program }\end{array}$ & Fallriver, MA & $\mathrm{n} / \mathrm{s}$ & January 200I - March 200I \\
\hline Martinez, $2012^{51}$ & MMTP & New York, NY & Effectiveness & July 2006 - June 2008 \\
\hline Masson, $2013^{52}$ & MMTP & New York, NY & Efficacy & $2008-2011$ \\
\hline McGovern, $2006^{54}$ & Correctional facility & $\mathrm{n} / \mathrm{s}, \mathrm{MA}$ & $\mathrm{n} / \mathrm{s}$ & November 200I - May 2004 \\
\hline Morano, $2014^{57}$ & Mobile medical clinic & New Haven, CT & $\mathrm{n} / \mathrm{s}$ & $2012-2013$ \\
\hline Rifai, $2006^{61}$ & $\begin{array}{l}\text { VA } 28 \text {-day inpatient substance- } \\
\text { use treatment program }\end{array}$ & Rural Virginia & $\mathrm{n} / \mathrm{s}$ & January 2000 - October 200I \\
\hline White, $2016^{58}$ & $E D^{d}$ & Oakland, CA & $\mathrm{n} / \mathrm{s}$ & April 2014 - October 2014 \\
\hline
\end{tabular}

Note: PRECIS- 2 domain scale: I = full efficacy, 2 = partial efficacy, 3 = neither efficacy nor effectiveness, 4 = partial effectiveness, 5 = full effectiveness. ${ }^{2}$ Veterans Affairs; ${ }^{\mathrm{b}}$ methadone maintenance treatment program; 'sexually transmitted disease; ${ }^{\mathrm{d}}$ emergency department.

Abbreviations: STD, sexually transmitted disease; MMTP, methadone-maintenance-treatment program; VA, Veterans Affairs; ED, emergency department. 


\begin{tabular}{|c|c|c|c|c|c|c|c|c|c|}
\hline \multicolumn{10}{|c|}{ PRECIS-II Domain } \\
\hline $\begin{array}{l}\text { Eligibility } \\
\text { criteria }\end{array}$ & Recruitment & Setting & Organization & $\begin{array}{l}\text { Flexibility of } \\
\text { intervention } \\
\text { delivery }\end{array}$ & $\begin{array}{l}\text { Flexibility of } \\
\text { intervention } \\
\text { adherence }\end{array}$ & $\begin{array}{l}\text { Follow- } \\
\text { up }\end{array}$ & Outcome & Analysis & $\begin{array}{l}\text { Composite } \\
\text { score (SD) }\end{array}$ \\
\hline 4 & 4 & 4 & 5 & 5 & 5 & 5 & 5 & 5 & $4.67(0.50)$ \\
\hline 5 & 5 & 5 & 4 & 5 & 5 & 5 & 5 & 5 & $4.89(0.33)$ \\
\hline 2 & 5 & 5 & 3 & 4 & 4 & 4 & 3 & 2 & $3.56(1.13)$ \\
\hline 3 & 5 & 3 & 2 & 4 & 3 & 5 & 2 & 1 & $3.11(1.36)$ \\
\hline 5 & 5 & 5 & 3 & 3 & 3 & 3 & 4 & 4 & $3.89(0.93)$ \\
\hline 5 & 5 & 5 & 5 & 5 & 5 & 5 & 5 & 5 & $5.00(0.00)$ \\
\hline 5 & 4 & 5 & 5 & 5 & 5 & 5 & 5 & 5 & $4.89(0.33)$ \\
\hline 2 & 5 & 3 & 5 & I & I & 5 & 2 & 2 & $2.89(1.69)$ \\
\hline 5 & 5 & 5 & 5 & 4 & 4 & 5 & 5 & 2 & $4.44(1.01)$ \\
\hline 5 & 5 & 5 & 4 & 5 & 3 & 4 & 5 & 5 & $4.56(0.73)$ \\
\hline 5 & 5 & 5 & 5 & 5 & 5 & 5 & 5 & 5 & $5.00(0.00)$ \\
\hline 4 & 4 & 5 & 4 & 5 & 4 & 5 & 5 & 5 & $4.56(0.53)$ \\
\hline 2 & 1 & 5 & 1 & I & I & I & 3 & 2 & $1.89(1.36)$ \\
\hline 2 & 1 & 5 & 1 & 1 & 1 & 1 & 2 & 3 & $1.89(1.36)$ \\
\hline 5 & 5 & 5 & 3 & 5 & 5 & 5 & 5 & 5 & $4.78(0.67)$ \\
\hline 2 & 5 & 3 & 3 & 3 & 2 & 2 & 3 & 3 & $2.89(0.93)$ \\
\hline
\end{tabular}

\begin{tabular}{|c|c|c|c|c|c|c|c|c|c|}
\hline \multicolumn{10}{|c|}{ PRECIS-II Domain } \\
\hline $\begin{array}{l}\text { Eligibility } \\
\text { criteria }\end{array}$ & $\begin{array}{l}\text { Recruitment } \\
\text { path }\end{array}$ & Setting & Organization & $\begin{array}{l}\text { Flexibility of } \\
\text { intervention } \\
\text { delivery }\end{array}$ & $\begin{array}{l}\text { Flexibility of } \\
\text { intervention } \\
\text { adherence }\end{array}$ & $\begin{array}{l}\text { Follow- } \\
\text { up }\end{array}$ & Outcome & Analysis & $\begin{array}{l}\text { Composite } \\
\text { score (SD) }\end{array}$ \\
\hline 2 & 3 & 4 & 5 & 4 & 5 & 4 & 5 & 5 & $4.11(1.05)$ \\
\hline 4 & 4 & 5 & 5 & 3 & 5 & 3 & 4 & 4 & $4.11(0.78)$ \\
\hline 5 & 5 & 5 & 5 & 5 & 5 & 5 & 5 & 5 & $5.00(0.00)$ \\
\hline 2 & 5 & 5 & 5 & 3 & 3 & 3 & 2 & 2 & $3.33(1.32)$ \\
\hline 3 & 5 & 3 & 2 & 4 & 4 & 5 & 2 & 2 & $3.33(1.22)$ \\
\hline 5 & 4 & 5 & 5 & 5 & 5 & 5 & 5 & 5 & $4.89(0.33)$ \\
\hline 5 & 5 & 5 & 3 & 4 & 4 & 4 & 3 & 3 & $4.00(0.87)$ \\
\hline 5 & 5 & 5 & 4 & 5 & 4 & 4 & 5 & 5 & $4.67(0.50)$ \\
\hline 5 & 4 & 5 & 4 & 4 & 4 & 5 & 3 & 3 & $4.11(0.78)$ \\
\hline 5 & 5 & 5 & 3 & 5 & 5 & 5 & 5 & 5 & $4.78(0.67)$ \\
\hline 3 & 5 & 3 & 2 & 2 & 2 & 4 & 2 & 2 & $2.78(1.09)$ \\
\hline 2 & 2 & 3 & 2 & 3 & 2 & 2 & 3 & 3 & $2.44(0.53)$ \\
\hline
\end{tabular}


Table 3 PRECIS-2 assessments for treatment outcome $(n=23)$

\begin{tabular}{|c|c|c|c|c|c|}
\hline $\begin{array}{l}\text { First author, } \\
\text { publication year }\end{array}$ & Recruitment site & Location & $\begin{array}{l}\text { Stated study } \\
\text { design }\end{array}$ & Time period & $\begin{array}{l}\text { Treatment } \\
\text { drug licensed } \\
\text { or unlicensed }\end{array}$ \\
\hline Adeyemi, $2004^{62}$ & Viral hepatitis clinic & Cook County, IL & $\mathrm{n} / \mathrm{s}^{\mathrm{a}}$ & July 200I - December 2002 & Licensed \\
\hline Allen, $2003^{64}$ & Correctional facility & Cranston, RI & Efficacy & $1997-200 \mid$ & Licensed \\
\hline Bonkovsky, $2008^{63}$ & MMTP' & $\mathrm{n} / \mathrm{s}$ & Efficacy & $\mathrm{n} / \mathrm{s}$ & Licensed \\
\hline Bruce, $2012^{65}$ & MMTP & New Haven, CT & $\mathrm{n} / \mathrm{s}$ & $2007-2010$ & Licensed \\
\hline Butt, $2016^{66}$ & $\mathrm{VA}^{\mathrm{c}}$ & $\begin{array}{l}\text { Multiple VA sites } \\
\text { throughout USA }\end{array}$ & Efficacy & $\begin{array}{l}\text { October I 200I - June } 30 \\
2015\end{array}$ & Licensed \\
\hline Gunn, $2005^{44}$ & $\begin{array}{l}\text { Nonresidential } \\
\text { alternative sentencing } \\
\text { drug rehabilitation }\end{array}$ & San Diego, CA & $\mathrm{n} / \mathrm{s}$ & April 1999 - December 2002 & Licensed \\
\hline Harris, $2010^{45}$ & MMTP & New York, NY & $\mathrm{n} / \mathrm{s}$ & July 2003 - July 2005 & Licensed \\
\hline $\mathrm{Ho}, 2013^{67}$ & $\begin{array}{l}\text { Primary care co- } \\
\text { located in a homeless } \\
\text { shelter }\end{array}$ & San Jose, CA & $\mathrm{n} / \mathrm{s}$ & $\mathrm{n} / \mathrm{s}$ & Licensed \\
\hline $\mathrm{Ho}, 2015^{68}$ & VA HCV clinic & $\begin{array}{l}\text { San Diego, CA; Palo } \\
\text { Alto, CA; New York, } \\
\text { NY }\end{array}$ & $\mathrm{n} / \mathrm{s}$ & March 2009 - February 2011 & Licensed \\
\hline Lalezari, $2015^{69}$ & $\mathrm{MAT}^{\mathrm{d}}$ clinic & 8 sites across the USA & Efficacy & April $2013-\mathrm{n} / \mathrm{s}$ & Unlicensed \\
\hline Litwin, $2009^{70}$ & MMTP & New York, NY & $\mathrm{n} / \mathrm{s}$ & $\begin{array}{l}\text { January I } 2003 \text { - December } \\
\text { I5 } 2005\end{array}$ & Licensed \\
\hline Litwin, $2015^{71}$ & MAT clinic & New York, NY & $\mathrm{n} / \mathrm{s}$ & January 212011 - April 22013 & Licensed \\
\hline Martinez, $2012^{51}$ & Viral hepatitis clinic & New York, NY & Effectiveness & July 2006 - June 2008 & Licensed \\
\hline Mehta, $2006^{72}$ & HIV clinic & Baltimore, MD & Effectiveness & June 1998 - December 2003 & Licensed \\
\hline Miller, $2012^{73}$ & Liver clinic & Atlanta, GA & $\mathrm{n} / \mathrm{s}$ & $2002-2007$ & Licensed \\
\hline Nelson, $2014^{74}$ & VA & Entire USA & Efficacy & $\begin{array}{l}\text { January I } 2004 \text { - December } \\
\text { 31 } 2009\end{array}$ & Licensed \\
\hline Rifai, $2006^{61}$ & $\begin{array}{l}\text { VA 28-day inpatient } \\
\text { substance-use } \\
\text { treamtent program }\end{array}$ & Rural Virginia & $\mathrm{n} / \mathrm{s}$ & January 2000 - October 2001 & Licensed \\
\hline Stein, $2012^{75}$ & MMTP & New York, NY & Effectiveness & March 2009 - October 2010 & Licensed \\
\hline Sylvestre, $2002^{76}$ & MMTP & Oakland, CA & Efficacy & $\mathrm{n} / \mathrm{s}$ & Licensed \\
\hline Sylvestre, $2005^{77}$ & MMTP & $\begin{array}{l}\text { Oakland, CA and New } \\
\text { York, NY }\end{array}$ & $\mathrm{n} / \mathrm{s}$ & $\mathrm{n} / \mathrm{s}$ & Licensed \\
\hline Taylor, $2011^{78}$ & MMTP & Providence, RI & Efficacy & $\mathrm{n} / \mathrm{s}$ & Licensed \\
\hline Van Thiel, $2003^{79}$ & Hospital clinic & Maywod, IL & $\mathrm{n} / \mathrm{s}$ & August 1997 - December 2000 & Licensed \\
\hline Woodrell, $2015^{80}$ & $\begin{array}{l}\text { Primary care liver } \\
\text { clinic }\end{array}$ & New York, NY & $\mathrm{n} / \mathrm{s}$ & $2011-2013$ & Licensed \\
\hline
\end{tabular}

Note: PRECIS-2 domain scale: I = full efficacy, 2 = partial efficacy, 3 = neither efficacy nor effectiveness, 4 = partial effectiveness, 5 = full effectiveness. aNot specified; bmethadone maintenance treatment program; 'Veterans Affairs; ${ }^{\mathrm{d}}$ medication assisted treatment.

Abbreviations: $\mathrm{n} / \mathrm{s}$, not specified; MMTP, methadone-maintenance-treatment program; VA, Veterans Affairs; HCV, hepatitis C virus; MAT, medication-assisted treatment.

on an efficacy-effectiveness spectrum studies identified and included in this systematic review of outcomes of the steps of the HCV-care continuum lie. ${ }^{84}$ We have now applied this adapted instrument in the context of this systematic review of outcomes of the steps of the HCV-care continuum. ${ }^{31}$

There was significant heterogeneity in the distribution of efficacy- and effectiveness-study design elements among reports examining the $\mathrm{HCV}$-care continuum identified in this systematic review. The majority of reports did not specify whether the study was intended to examine efficacy or effectiveness. This might reflect a recognition that study designs exist along a spectrum of efficacy and effectiveness, but it might also reflect incomplete consideration of these issues at the time of study design. As highlighted by Loudon et al, ${ }^{39}$ use of the PRECIS-2 instrument may be invaluable during the process of study planning to help ensure that investigators select study-design elements that most appropriately answer the study question, whether that be one of efficacy, effectiveness, or some combination.

The PRECIS-2 toolkit gives guidance on how to score each domain, and rather than suggesting a composite score, suggests scores be depicted visually in a wheel. ${ }^{8}$ 


\begin{tabular}{|c|c|c|c|c|c|c|c|c|c|}
\hline \multicolumn{10}{|c|}{ PRECIS-II Domain } \\
\hline $\begin{array}{l}\text { Eligibility } \\
\text { criteria }\end{array}$ & $\begin{array}{l}\text { Recruitment } \\
\text { path }\end{array}$ & Setting & Organization & $\begin{array}{l}\text { Flexibility of } \\
\text { intervention } \\
\text { adherence }\end{array}$ & $\begin{array}{l}\text { Flexibility of } \\
\text { intervention } \\
\text { adherence }\end{array}$ & Follow-up & Outcome & Analysis & $\begin{array}{l}\text { Score } \\
\text { (SD) }\end{array}$ \\
\hline 2 & 5 & 4 & 5 & 4 & 4 & 4 & 4 & 4 & $4.00(0.87)$ \\
\hline 4 & 5 & 5 & 5 & 5 & 4 & 4 & 4 & 2 & $4.22(0.97)$ \\
\hline I & 3 & 5 & I & 1 & 1 & I & 2 & 2 & $1.89(1.36)$ \\
\hline 2 & 3 & 5 & 3 & I & I & 1 & I & I & $2.00(I .4 I)$ \\
\hline I & 3 & 4 & 3 & 2 & 2 & 3 & 2 & 2 & $2.44(0.88)$ \\
\hline 5 & 5 & 5 & 5 & 5 & 5 & 5 & 5 & 5 & $5.00(0.00)$ \\
\hline 2 & 5 & 5 & 2 & 2 & 3 & 3 & 2 & I & $2.78(1.39)$ \\
\hline 3 & 4 & 5 & 3 & 3 & 2 & 4 & 2 & 2 & $3.11(1.05)$ \\
\hline 3 & 3 & 4 & 3 & 2 & 3 & 3 & 4 & 3 & $3.11(0.60)$ \\
\hline 3 & I & I & 1 & 1 & I & I & I & I & $1.22(0.67)$ \\
\hline 2 & 5 & 3 & 5 & 2 & 2 & 2 & 5 & 2 & $3.11(1.45)$ \\
\hline 3 & 5 & 5 & 3 & 4 & 4 & 4 & 5 & 3 & $4.00(0.87)$ \\
\hline 5 & 5 & 5 & 3 & 4 & 4 & 3 & 3 & 2 & 3.78 (1.09) \\
\hline 4 & 4 & 5 & 5 & 3 & 5 & 3 & 4 & 3 & $4.00(0.87)$ \\
\hline 2 & 2 & 2 & 3 & 2 & 4 & 4 & 2 & 4 & $2.78(0.97)$ \\
\hline 2 & 5 & 5 & 4 & 2 & 4 & 2 & 4 & 4 & $3.56(1.24)$ \\
\hline 2 & 3 & 5 & 4 & I & I & I & 2 & I & $2.22(1.48)$ \\
\hline 5 & 3 & 3 & 5 & 4 & 3 & 3 & 3 & 4 & $3.67(0.87)$ \\
\hline 2 & 5 & 3 & 3 & 2 & 3 & 4 & 2 & 2 & $2.89(1.05)$ \\
\hline 3 & 5 & 5 & 4 & 3 & 3 & 4 & 4 & 3 & $3.78(0.83)$ \\
\hline 3 & 5 & 5 & 3 & 3 & 3 & 3 & 5 & 4 & $3.78(0.97)$ \\
\hline 3 & 5 & 4 & 3 & 2 & 3 & 2 & 3 & 4 & $3.22(0.97)$ \\
\hline 5 & 5 & 5 & 5 & 5 & 5 & 5 & 5 & 5 & $5.00(0.00)$ \\
\hline
\end{tabular}

Wheel charts do serve as potent infographics representing the degrees of heterogeneity or homogeneity found in the efficacy-effectiveness design of studies. As demonstrated by the heterogeneity in efficacy-effectiveness study design identified among the included reports, studies of any given care-continuum step can vary significantly in efficacy-effectiveness design and in their positions on the efficacy-effectiveness spectrum. As seen in Figures 2 and 3, for a given outcome and even within a single publication examining all three HCV-care-continuum steps, the balance of efficacyeffectiveness study-design elements may vary.
Studies of HCV testing and linkage to care had mean scores for all nine domains that tended toward effectiveness, particularly for the domains of setting, recruitment path, and follow-up. For testing studies, this is consistent with the fact that throughout the period studied in the systematic review, HCV-diagnostic tests of high sensitivity and specificity have been available and key questions have been on how to engage more PWUD in testing. While behavioral and structural interventions to promote linkage to care could in theory be evaluated to assess their efficacy or for questions of effectiveness, linkage to care reports identified in this review 
Outcomes:

Testing
Linkage to care
Treatment

One outcome scored

Two or three outcomes shared score

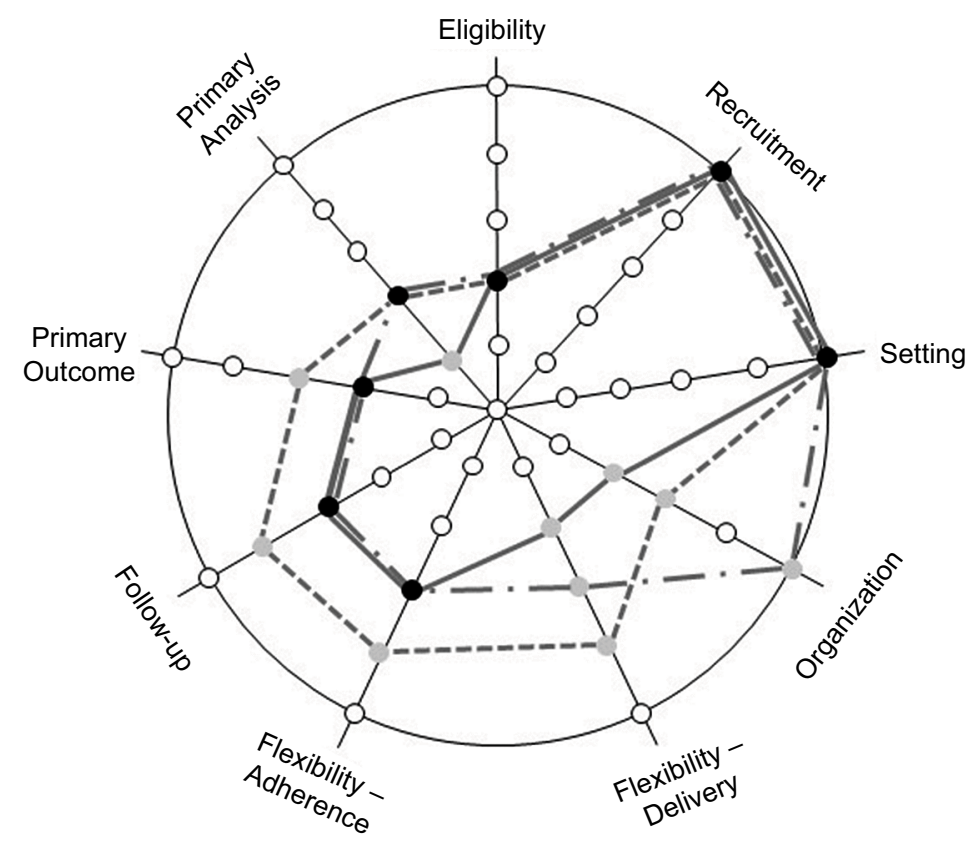

Figure 2 PRECIS-2 wheel chart for three outcomes in Harris et al. ${ }^{45}$

tended to have effectiveness-study designs, as appropriate in a situation in which the efficacy of testing and treatment have been well demonstrated. ${ }^{44,49,52}$

It is notable that mean PRECIS-2 composite scores for treatment studies were significantly lower than for those examining testing or linkage to care, despite the fact that all except one of the treatment studies were studies of already licensed treatment regimens. While treatment studies tended modestly toward effectiveness designs overall, and especially with respect to recruitment path and study setting, they tended toward efficacy designs with respect to eligibility, the flexibility of delivery, and analysis. Reasons for this are uncertain; however, one possibility is a "carryover" to studies of licensed drugs of the study-design methods appropriately employed in the evaluation of investigational drugs. The tendency toward effectiveness-study design with respect to study setting and recruitment path may reflect a recognition of the need to engage PWUD in settings they attend. Use of efficacy-design elements may have been prompted by efforts to examine whether HCV-treatment outcomes among PWUD can be as a good in idealized conditions as among non-PWUD. These data may have been needed to influence policy-makers to include PWUD in treatment guidelines. ${ }^{85,86}$ Addressing the other critical question of how good HCV-treatment outcomes among PWUD are in routine care and at the population level may be more appropriately examined and addressed by use of effectiveness-study designs.

While wheel charts have been utilized in reviews of modest numbers of publications, ${ }^{36,37}$ they lose their visual clarity and potency as the number of reviewed reports increases. It becomes impractical either to depict each paper separately or as superimposed wheel charts. We thus sought alternative ways to present PRECIS-2 score data visually for larger numbers of studies. We used stacked dot plots to represent the distribution of scores for each domain for each paper included in a systematic review. We also represented scores by their means (with standard deviations) in tabular form. These formats may be useful approaches for other studies applying the PRECIS-2 instrument to large numbers of reports.

Several issues arise in incorporating measures of a study's efficacy-effectiveness status in systematic reviews/metaanalyses. Dichotomous assessments, such as that of Gartlehner et al, yield a dichotomous variable that is easily incorporated in meta-analyses. ${ }^{81}$ However, as demonstrated by the heterogeneity of efficacy-effectiveness study designs identified among the included reports (see Tables 2-4), papers in this systematic review are more richly and accurately categorized along a spectrum of efficacy-effectiveness in numerous domains.

The use of a measure with nine domains, each scored with a 5-point Likert-like scale, poses choices as to the best way 
A

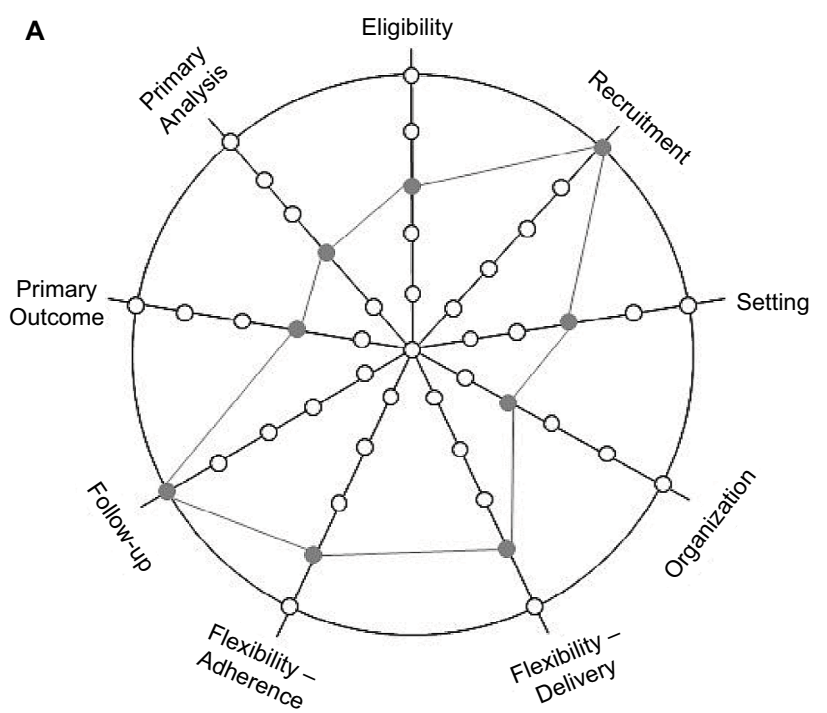

B
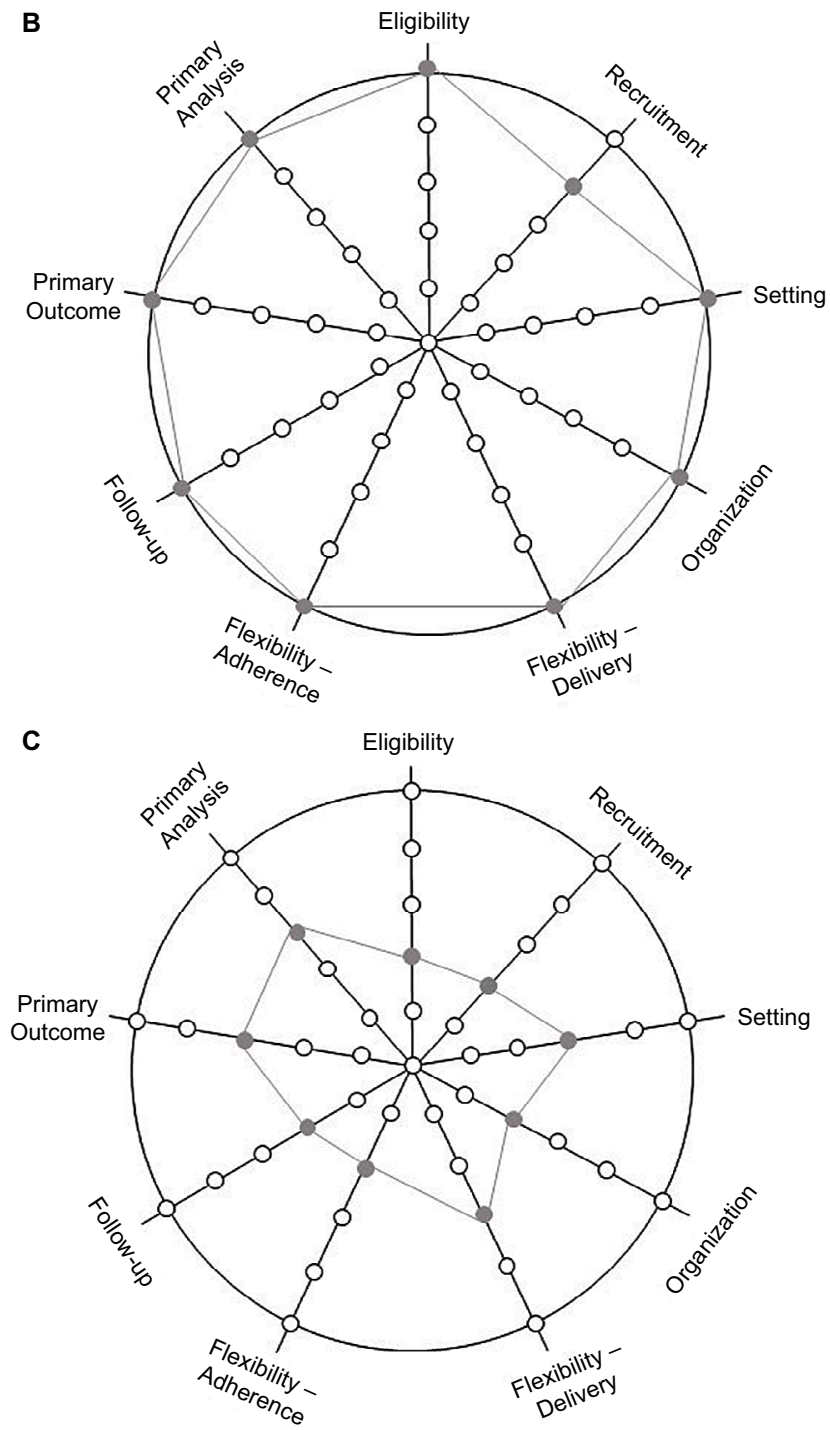

Figure 3 PRECIS-2 wheel charts.

Notes: (A) Linkage to care outcome in Hennessy et $\mathrm{a}^{46}$; (B) linkage to care outcome in Lally et a a ${ }^{49}$; (C) linkage to care outcome in White et al. ${ }^{58}$ to incorporate these measures of efficacy-effectiveness study design into meta-analyses. One could consider each domain a separate variable with a range of $1-5$, and examine each domain as a covariate in metaregression. If one particular domain were found to differentiate included studies more potently, one could examine that domain exclusively in metaanalysis, as opposed to incorporating all nine domains as covariates. A study would have to include a sufficient number of papers to examine nine separate efficacy-effectiveness domains as covariates. Some studies have utilized composite PRECIS-2 scores for each report in metaregression, rather than examining each domain as a distinct covariate. ${ }^{30,37}$

This study is subject to limitations. One limitation is that the systematic review examined only reports that specifically included PWUD and that reported data for PWUD separately. We note that many studies of HCV may include PWUD without explicitly saying so or without reporting data separately for PWUD as a group (and hence would not be included in this systematic review), and also note that many studies (particularly treatment-randomized controlled trials) may specifically exclude PWUD. Nonetheless, the intent of the systematic review was to examine data specifically for the HCV-care continuum among PWUD as a distinct population. The rationale for the focus on this population is that PWUD as a population have distinct characteristics impacting outcomes and thus require analysis as a separate group. ${ }^{32}$ One limitation was that reports were coded by a single rater, with scores then reviewed by the study team; however, the PRECIS-2 instrument has been shown to have good interrater reliability. ${ }^{39}$ Numerous studies did not either specify their recruitment time periods or present data that spanned multiple years, precluding sound analyses of any temporal trends. The level of data provided in the included reports with respect to some of the domains was variable, and could have contributed to misclassification of studies with respect to PRECIS-2 scores.

\section{Conclusion}

The heterogeneity identified in efficacy-effectiveness study design suggests that variability needs to be accounted for in systematic reviews and meta-analyses of the HCV-care continuum. Use of an adapted PRECIS-2 instrument with scores depicted in stacked dot plots and in tabular form will help guide how to incorporate these data in meta-analysis. Studies presenting outcomes of interventions to improve the steps of the HCV-care continuum would be enhanced by more clearly framing their study design and results with respect 


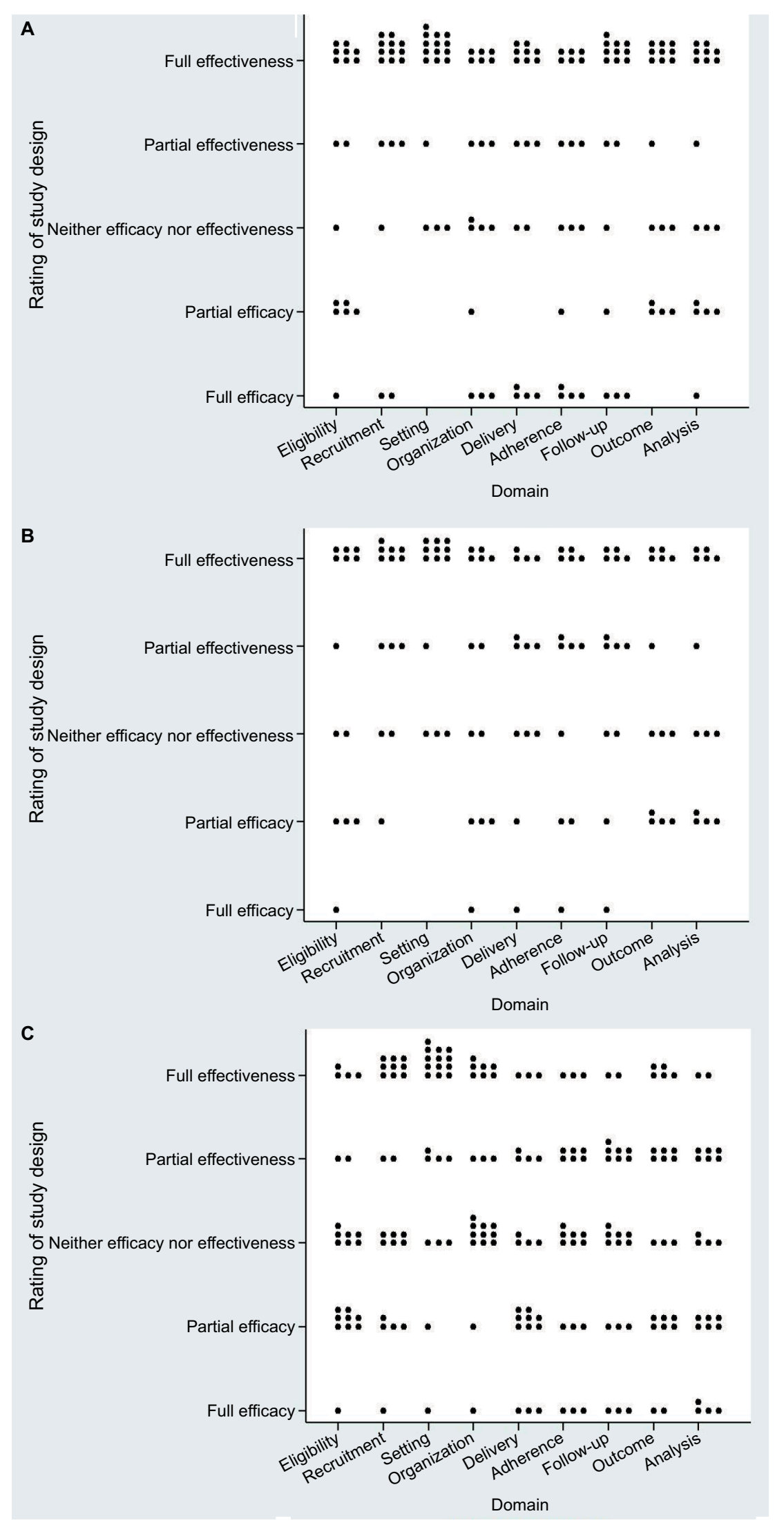

Figure 4 (A) HCV-testing outcome; (B) HCV linkage to care outcome; (C) HCV-treatment outcome. Abbreviation: $\mathrm{HCV}$, hepatitis $\mathrm{C}$ virus. 
Table 4 Composite score results from PRECIS-2 assessment for 37 reports in HCV-care-continuum systematic review

\begin{tabular}{|c|c|c|c|c|c|c|}
\hline \multirow{3}{*}{$\begin{array}{l}\text { PRECIS-II Domains }(\mathrm{n}=9) \\
\text { Eligiblity }\end{array}$} & \multicolumn{2}{|c|}{$\begin{array}{l}\text { Testing } \\
(n=16 \text { outcomes) }\end{array}$} & \multirow{2}{*}{\multicolumn{2}{|c|}{$\begin{array}{l}\text { Linkage to care } \\
\text { ( } \mathrm{n}=\mathrm{I} 2 \text { outcomes) } \\
\text { Mean (SD) Median }\end{array}$}} & \multicolumn{2}{|c|}{$\begin{array}{l}\text { Treatment } \\
\text { ( } n=23 \text { outcomes) }\end{array}$} \\
\hline & \multirow{2}{*}{$\begin{array}{l}\text { Mean (SD) } \\
3.81(1.50)\end{array}$} & \multirow{2}{*}{$\begin{array}{l}\text { Median } \\
4.50\end{array}$} & & & Mean (SD) & Median \\
\hline & & & $3.83(1.50)$ & 4.50 & $2.91(1.20)$ & 3.00 \\
\hline Recruitment path & $4.31(1.35)$ & 5.00 & $4.33(1.01)$ & 5.00 & $4.09(1.21)$ & 5.00 \\
\hline Setting & $4.56(0.80)$ & 5.00 & $4.42(0.88)$ & 5.00 & $4.26(1.15)$ & 5.00 \\
\hline Organization & $3.63(1.50)$ & 4.00 & $3.75(1.45)$ & 4.00 & $3.52(1.14)$ & 3.00 \\
\hline Flexibility of delivery & $3.81(1.66)$ & 4.50 & $3.92(1.25)$ & 4.00 & $2.74(1.30)$ & 2.00 \\
\hline Flexibility of adherence & $3.50(1.62)$ & 4.00 & $4.00(1.36)$ & 4.00 & $3.04(1.25)$ & 3.00 \\
\hline Follow-up & $4.06(1.62)$ & 5.00 & $4.08(1.28)$ & 4.00 & $3.00(1.19)$ & 3.00 \\
\hline Outcome & $4.00(1.32)$ & 5.00 & $3.67(1.33)$ & 3.50 & $3.22(1.35)$ & 3.00 \\
\hline Analysis & $3.69(1.46)$ & 4.50 & $3.67(1.33)$ & 3.50 & $2.78(1.30)$ & 3.00 \\
\hline $\begin{array}{l}\text { Mean (SD) PRECIS-II summary score } \\
\text { by outcome }\end{array}$ & $3.85(0.34)$ & - & $3.80(0.27)$ & - & $3.35(0.56)$ & - \\
\hline
\end{tabular}

Note: PRECIS-2 domain scale: I = full efficacy, 2 = partial efficacy, 3 = neither efficacy nor effectiveness, 4 = partial effectiveness, $5=$ full effectiveness.

Abbreviation: $\mathrm{HCV}$, hepatitis $\mathrm{C}$ virus.

to the extent to which they are measuring and reporting on efficacy or effectiveness. This review of intervention studies examining the $\mathrm{HCV}$-care continuum found that most study designs tended toward effectiveness designs, consistent with the recognized still-existing population-level gap between the efficacy of testing, linkage, and treatment interventions and the current population-level net effectiveness of HCV-care systems in moving individuals and populations through the HCV-care continuum to SVR and cure. Treatment studies, nonetheless, were more likely to utilize efficacy study-design elements, particularly in the domains of eligibility, flexibility of delivery, and analysis. This is notable, given the solid evidence of the efficacy of current HCV-treatment agents. It suggests that one important gap in the literature may be the need for more data on the population-level effectiveness of $\mathrm{HCV}$ treatment in broader populations of PWUD treated in appropriate but routine models of care.

\section{Acknowledgments}

The HCV Synthesis Project is supported by a grant from the National Institutes of Health (RO1DA034637-01). Support was also received from the New York University Center for Drug Use and HIV Research, an NIH P30 center (P30 DA011041).

\section{Disclosure}

The authors report no conflicts of interest in this work.

\section{References}

1. Carey T, Yon A, Beadles C, Wines R. Prioritizing future research through examination of research gaps in systematic reviews. 2012. Available from: https://www.pcori.org/assets/Prioritizing-Future-Researchthrough-Examination-of-Research-Gaps-in-Systematic-Reviews1.pdf. Accessed February 3, 2018.
2. Miake-Lye IM, Hempel S, Shanman R, Shekelle PG. What is an evidence map? A systematic review of published evidence maps and their definitions, methods, and products. Syst Rev. 2016;5:28.

3. Chalmers I, Fox DM. Increasing the incidence and influence of systematic reviews on health policy and practice. Am J Public Health. 2016;106(1):11-13.

4. Singal AG, Higgins PD, Waljee AK. A primer on effectiveness and efficacy trials. Clin Transl Gastroenterol. 2014;5:e45.

5. Osterholm MT, Kelley NS, Sommer A, Belongia EA. Efficacy and effectiveness of influenza vaccines: a systematic review and meta-analysis. Lancet Infect Dis. 2012;12(1):36-44.

6. Umscheid CA. A primer on performing systematic reviews and metaanalyses. Clin Infect Dis. 2013;57(5):725-734.

7. Wells KB. Treatment research at the crossroads: the scientific interface of clinical trials and effectiveness research. Am JPsychiatry. 1999;156(1):5-10.

8. Loudon K, Treweek S, Sullivan F, Donnan P, Thorpe KE, Zwarenstein M. The PRECIS-2 tool: designing trials that are fit for purpose. $B M J$. 2015;350:h2147.

9. Althuis MD, Weed DL, Frankenfeld CL. Evidence-based mapping of design heterogeneity prior to meta-analysis: a systematic review and evidence synthesis. Syst Rev. 2014;3:80

10. Karanicolas PJ, Montori VM, Devereaux PJ, Schunemann H, Guyatt GH. A new "mechanistic-practical" framework for designing and interpreting randomized trials. J Clin Epidemiol. 2009;62(5):479-484.

11. Solomon SS, Mehta SH, Srikrishnan AK, et al. Burden of hepatitis $\mathrm{C}$ virus disease and access to hepatitis $\mathrm{C}$ virus services in people who inject drugs in India: a cross-sectional study. Lancet Infect Dis. 2015;15(1):36-45.

12. Smith DJ, Combellick J, Jordan AE, Hagan H. Hepatitis C virus (HCV) disease progression in people who inject drugs (PWID): a systematic review and meta-analysis. Int J Drug Policy. 2015;26(10):911-921.

13. Ford N, Wiktor S, Kaplan K, et al. Ten priorities for expanding access to HCV treatment for people who inject drugs in low- and middle-income countries. Int J Drug Policy. 2015;26(11):1088-1093.

14. Wolfe D, Luhmann N, Harris M, et al. Human rights and access to hepatitis C treatment for people who inject drugs. Int J Drug Policy. 2015;26(11):1072-1080.

15. Hagan H, Latka MH, Campbell JV, et al. Eligibility for treatment of hepatitis $\mathrm{C}$ virus infection among young injection drug users in 3 US cities. Clin Infect Dis. 2006;42(5):669-672.

16. Corson S, Greenhalgh D, Palmateer N, Weir A, Hutchinson S. Risk of Hepatitis $\mathrm{C}$ virus re-infection following spontaneous viral clearance in injecting drug users: a systematic review. Int J Drug Policy. 2011;22(2):102-108. 
17. Perlman DC, des Jarlais DC, Feelemyer J. Can HIV and hepatitis C virus infection be eliminated among persons who inject drugs? J Addict Dis. 2015;34(2-3):198-205.

18. Trooskin SB, Reynolds H, Kostman JR. Access to costly new hepatitis c drugs: medicine, money, and advocacy. Clin Infect Dis. 2015;61(12): 1825-1830.

19. Turner K, Hutchinson S, Vickerman P, et al. The impact of needle and syringe provision and opiate substitution therapy on the incidence of hepatitis $\mathrm{C}$ virus in injecting drug users: pooling of UK evidence. Addiction. 2011;106(11):1978-1988.

20. Hagan H, Pouget ER, des Jarlais DC. A systematic review and metaanalysis of interventions to prevent hepatitis $\mathrm{C}$ virus infection in people who inject drugs. J Infect Dis. 2011;204(1):74-83.

21. Martin NK, Vickerman P, Miners A, et al. Cost-effectiveness of hepatitis $\mathrm{C}$ virus antiviral treatment for injection drug user populations. Hepatology. 2012;55(1):49-57.

22. Martin NK, Vickerman P, Grebely J, et al. Hepatitis C virus treatment for prevention among people who inject drugs: modeling treatment scale-up in the age of direct-acting antivirals. Hepatology. 2013;58(5):1598-1609.

23. Hagan H, Pouget R, des Jarlais DC. A systematic review and metaanalysis of interventions to prevent hepatitis $\mathrm{C}$ virus infection in people who inject drugs. J Infect Dis. 2011 1;204(1):74-83.

24. van Handel MM, Rose CE, Hallisey EJ, et al. County-level vulnerability assessment for rapid dissemination of HIV or HCV infections among persons who inject drugs, United States. JAcquir Immune Defic Syndr. 2016;73(3):323-331.

25. Perlman DC, Jordan AE, Nash D. Conceptualizing care continua: lessons from HIV, hepatitis C Virus, tuberculosis and implications for the development of improved care and prevention continua. Front Public Health. 2017;4:296.

26. Linas BP, Barter DM, Leff JA, et al. The hepatitis C cascade of care: identifying priorities to improve clinical outcomes. PLoS One. 2014;9(5):e97317.

27. Yu ML, Yeh ML, Tsai PC, et al. Huge gap between clinical efficacy and community effectiveness in the treatment of chronic hepatitis C: a nationwide survey in Taiwan. Medicine (Baltimore). 2015;94(13):e690.

28. Haley SJ, Kreek MJ. A window of opportunity: maximizing the effectiveness of new HCV regimens in the United States with the expansion of the Affordable Care Act. Am J Public Health. 2015;105(3):457-463.

29. Kramer JR, Kanwal F, Richardson P, Mei M, El-Serag HB. Gaps in the achievement of effectiveness of HCV treatment in national VA practice. $J$ Hepatol. 2012;56(2):320-325.

30. Kaner E, Beyer F, Dickinson H, et al. Effectiveness of brief alcohol interventions in primary care populations. Cochrane Database Syst Rev. 2007;(2):CD004148.

31. Reed JR, Jordan AE, Perlman DC, Smith DJ, Hagan H. The HCV care continuum among people who use drugs: protocol for a systematic review and meta-analysis. Syst Rev. 2016;5(1):110.

32. Krieger N. Who and what is a "population"? Historical debates, current controversies, and implications for understanding "population health" and rectifying health inequities. Milbank Q. 2012;90(4):634-681.

33. Mangal JP, Rimland D, Marconi VC. The continuum of HIV care in a Veterans' Affairs clinic. AIDS Res Hum Retroviruses. 2014;30(5): 409-415.

34. Ali MK, Bullard KM, Gregg EW, Del Rio C. A cascade of care for diabetes in the United States: visualizing the gaps. Ann Iintern Med. 2014;161(10):681-689.

35. Moher D, Shamseer L, Clarke M, et al. Preferred reporting items for systematic review and meta-analysis protocols (PRISMA-P) 2015 statement. Syst Rev. 2015;4:1.

36. Johnson KE, Neta G, Dember LM, et al. Use of PRECIS ratings in the National Institutes of Health $(\mathrm{NIH})$ Health Care Systems Research Collaboratory. Trials. 2016;17:32.

37. Cruvinel E, Richter KP, Stoney C, et al. CHARTing a path to pragmatic tobacco treatment research. Am J Prev Med. 2016;51(4):630-636.
38. Glasgow RE, Gaglio B, Bennett G, et al. Applying the PRECIS criteria to describe three effectiveness trials of weight loss in obese patients with comorbid conditions. Health Serv Res. 2012;47(3 Pt 1):1051-1067.

39. Loudon K, Zwarenstein M, Sullivan F, et al. The PRECIS-2 tool has good interrater reliability and modest discriminant validity. $J$ Clin Epidemiol. 2017;88:113-121.

40. PRECIS-2 [homepage on the internet]. 2016. Available from: http:// precis-2.org. Accessed July 5, 2017.

41. StataCorp. Stata statistical software: release 15. College Station (TX): StataCorp; 2017.

42. IBM. SPSS Statistics for Windows [software]. Armonk, NY: IBM; 2013.

43. D'Souza G, Arafat R, Hwang L, Cunningham C, Shah S, Reynolds $\mathrm{K}$. Cross-sectional survey of the extent and indicators of hepatitis $\mathrm{C}$ virus infection in Houston Department of Health and Human Services' sexually transmitted disease clinics. J Viral Hepat. 2003;10(2): $134-140$.

44. Gunn RA, Lee MA, Callahan DB, Gonzales P, Murray PJ, Margolis HS. Integrating hepatitis, STD, and HIV services into a drug rehabilitation program. Am J Prev Med. 2005;29(1):27-33.

45. Harris KA Jr, Arnsten JH, Litwin AH. Successful integration of hepatitis $\mathrm{C}$ evaluation and treatment services with methadone maintenance. $J$ Addict Med. 2010;4(1):20-26.

46. Hennessy RR, Weisfuse IB, Schlanger K. Does integrating viral hepatitis services into a public STD clinic attract injection drug users for care? Public Health Rep. 2007;122 Suppl 2:31-35.

47. Kapadia F, Latka MH, Hagan H, et al. Design and feasibility of a randomized behavioral intervention to reduce distributive injection risk and improve health-care access among hepatitis $\mathrm{C}$ virus positive injection drug users: the Study to Reduce Intravenous Exposures (STRIVE). J Urban Health. 2007;84(1):99-115.

48. Kim AY, Nagami EH, Birch CE, Bowen MJ, Lauer GM, McGovern BH. A simple strategy to identify acute hepatitis $\mathrm{C}$ virus infection among newly incarcerated injection drug users. Hepatology. 2013;57(3): 944-952.

49. Lally MA, MacNevin R, Sergie Z, et al. A model to provide comprehensive testing for HIV, viral hepatitis, and sexually transmitted infections at a short-term drug treatment center. AIDS Patient Care STDs. 2005;19(5):298-305.

50. Mallette C, Flynn MA, Promrat K. Outcome of screening for hepatitis $\mathrm{C}$ virus infection based on risk factors. Am J Gastroenterol. 2008;103(1):131-137.

51. Martinez A, Dimova R, Marks K, et al. Integrated internist - addiction medicine - hepatology model for hepatitis $\mathrm{C}$ management for individuals on methadone maintenance. J Viral Hepat. 2012;19(1):47-54.

52. Masson CL, Delucchi KL, McKnight C, et al. A randomized trial of a hepatitis care coordination model in methadone maintenance treatment. Am J Public Health. 2013;103(10):e81-e88.

53. McGarry KA, Stein MD, Clarke JG, Friedmann PD. Utilization of preventive health services by HIV-seronegative injection drug users. J Addict Dis. 2002;21(2):93-102.

54. McGovern BH, Wurcel A, Kim AY, et al. Acute hepatitis C virus infection in incarcerated injection drug users. Clin Infect Dis. 2006;42(12):1663-1670.

55. Merchant RC, Baird JR, Liu T, Taylor LE, Montague BT, Nirenberg TD. Brief intervention to increase emergency department uptake of combined rapid human immunodeficiency virus and hepatitis $\mathrm{C}$ screening among a drug misusing population. Acad Emerg Med. 2014;21(7): 752-767.

56. Merchant RC, DeLong AK, Liu T, Baird JR. Factors influencing uptake of rapid HIV and hepatitis $\mathrm{C}$ screening among drug misusing adult emergency department patients: implications for future $\mathrm{HIV} / \mathrm{HCV}$ screening interventions. AIDS Behav. 2015;19(11):2025-2035.

57. Morano J, Zelenev A, Lombard A, Marcus R, Gibson B, Altice F. Strategies for hepatitis $\mathrm{C}$ testing and linkage to care for vulnerable populations: point-of-care and standard HCV testing in a mobile medical clinic. J Community Health. 2014;39(5):922-934. 
58. White DA, Anderson ES, Pfeil SK, Trivedi TK, Alter HJ. Results of a rapid hepatitis $\mathrm{C}$ virus screening and diagnostic testing program in an urban emergency department. Ann Emerg Med. 2016;67(1):119-128.

59. Brady CW, Coffman CJ, Provenzale D. Compliance with referral for hepatitis C evaluation among veterans. J Clin Gastroenterol. 2007;41(10): 927-931.

60. Fishbein DA, Lo Y, Reinus JF, Gourevitch MN, Klein RS. Factors associated with successful referral for clinical care of drug users with chronic hepatitis $\mathrm{C}$ who have or are at risk for HIV infection. J Acquir Immune Defic Syndr. 2004;37(3):1367-1375.

61. Rifai MA, Moles JK, Lehman LP, van der Linden BJ. Hepatitis C screening and treatment outcomes in patients with substance use/dependence disorders. Psychosomatics. 2006;47(2):112-121.

62. Adeyemi OM, Jensen D, Attar B, et al. Hepatitis C treatment eligibility in an urban population with and without HIV coinfection. AIDS Patient Care STDS. 2004;18(4):239-245.

63. Bonkovsky HL, Tice AD, Yapp RG, et al. Efficacy and safety of PEGinterferon $-2 \mathrm{a} /$ ribavirin in methadone maintenance patients: randomized comparison of direct observed therapy and self-administration. Am J Gastroenterol. 2008;103(11):2757-2765.

64. Allen SA, Spaulding AC, Osei AM, Taylor LE, Cabral AM, Rich JD. Treatment of chronic hepatitis $\mathrm{C}$ in a state correctional facility. Ann Intern Med. 2003;138(3):187-190.

65. Bruce RD, Eiserman J, Acosta A, Gote C, Lim JK, Altice FL. Developing a modified directly observed therapy intervention for hepatitis $\mathrm{C}$ treatment in a methadone maintenance program: implications for program replication. Am J Drug Alcohol Abuse. 2012;38(3):206-212.

66. Butt AA, Yan P, Shaikh OS, Chung RT, Sherman KE. Sofosbuvir-based regimens in clinical practice achieve SVR rates closer to clinical trials: results from ERCHIVES. Liver Int. 2016;36(5):651-658.

67. Ho CJ, Preston C, Fredericks K, et al. A unique model for treating chronic hepatitis $\mathrm{C}$ in patients with psychiatric disorders, substance abuse, and/or housing instability. J Addict Med. 2013;7(5):320-324.

68. Ho SB, Brau N, Cheung R, et al. Integrated care increases treatment and improves outcomes of patients with chronic hepatitis $\mathrm{C}$ virus infection and psychiatric illness or substance abuse. Clin Gastroenterol Hepatol. 2015;13(11):2005-2014.e1-e3.

69. Lalezari J, Sullivan JG, Varunok P, et al. Ombitasvir/paritaprevir/r and dasabuvir plus ribavirin in HCV genotype 1-infected patients on methadone or buprenorphine. J Hepatol. 2015;63(2):364-369.

70. Litwin AH, Harris KA Jr, Nahvi S, et al. Successful treatment of chronic hepatitis $\mathrm{C}$ with PEGylated interferon in combination with ribavirin in a methadone maintenance treatment program. $J$ Subst Abuse Treat. 2009;37(1):32-40.

71. Litwin AH, Soloway IJ, Cockerham-Colas L, et al. Successful treatment of chronic hepatitis $\mathrm{C}$ with triple therapy in an opioid agonist treatment program. Int J Drug Policy. 2015;26(10):1014-1019.
72. Mehta SH, Lucas GM, Mirel LB, et al. Limited effectiveness of antiviral treatment for hepatitis C in an urban HIV clinic. AIDS. 2006;20(18): 2361-2369.

73. Miller L, Fluker SA, Osborn M, Liu X, Strawder A. Improving access to hepatitis $\mathrm{C}$ care for urban, underserved patients using a primary carebased hepatitis C clinic. J Natl Med Assoc. 2012;104(5-6):244-250.

74. Nelson RE, Hoop R, Korner E, et al. Predicting discontinuation of PEGylated interferon as a result of lack of efficacy in United States veterans with chronic hepatitis C on dual therapy. Ann Pharmacother. 2014;48(7): 826-836.

75. Stein MR, Soloway IJ, Jefferson KS, Roose RJ, Arnsten JH, Litwin $\mathrm{AH}$. Concurrent group treatment for hepatitis $\mathrm{C}$ : implementation and outcomes in a methadone maintenance treatment program. J Subst Abuse Treat. 2012;43(4):424-432.

76. Sylvestre DL. Treating hepatitis $C$ in methadone maintenance patients: an interim analysis. Drug Alcohol Depend. 2002;67(2):117-123.

77. Sylvestre DL, Litwin AH, Clements BJ, Gourevitch MN. The impact of barriers to hepatitis $\mathrm{C}$ virus treatment in recovering heroin users maintained on methadone. J Subst Abuse Treat. 2005;29(3):159-165.

78. Taylor LE, Bowman SE, Chapman S, et al. Treatment for hepatitis C virus genotype 1 infection in HIV-infected individuals on methadone maintenance therapy. Drug Alcohol Depend. 2011;116(1-3):233-237.

79. van Thiel DH, Anantharaju A, Creech S. Response to treatment of hepatitis $\mathrm{C}$ in individuals with a recent history of intravenous drug abuse. Am J Gastroenterol. 2003;98(10):2281-2288.

80. Woodrell C, Weiss J, Branch A, et al. Primary care-based hepatitis c treatment outcomes with first-generation direct-acting agents. J Addict Med. 2015;9(5):405-410.

81. Gartlehner G, Hansen RA, Nissman D, Lohr KN, Carey TS. A simple and valid tool distinguished efficacy from effectiveness studies. J Clin Epidemiol. 2006;59(10):1040-1048.

82. Gartlehner G, Hansen RA, Nissman D, Lohr KN, Carey TS. Criteria for Distinguishing Effectiveness from Efficacy Trials in Systematic Reviews. Rockville (MD): Agency for Healthcare Research and Quality; 2006.

83. Heather N. The efficacy-effectiveness distinction in trials of alcohol brief intervention. Addict Sci Clin Pract. 2014;9:13.

84. Jordan AE, Perlman DC, Smith DJ, Reed J, Hagan H. Use of the PRECIS-II instrument to categorize reports along the efficacy-effectiveness spectrum in an hepatitis $\mathrm{C}$ virus care continuum systematic review and meta-analysis. J Clin Epidemiol. 2018;93:66-75.

85. Hellard M, Sacks-Davis R, Gold J. Hepatitis C treatment for injection drug users: a review of the available evidence. Clin Infect Dis. 2009;49(4):561-573.

86. Edlin BR, Seal KH, Lorvick J, et al. Is it justifiable to withhold treatment for hepatitis C from illicit-drug users? N Engl J Med. 2001;345(3): $211-215$.
Comparative Effectiveness Research

\section{Publish your work in this journal}

Comparative Effectiveness Research is an international, peer reviewed open access journal focusing on comparative effectiveness of health care including preventative health care strategies, diagnostic strategies, diagnostic technology, medical devices, drugs, medical technology, health systems and organization. The manuscript management system

\section{Dovepress}

is completely online and includes a very quick and fair peer-review system. Visit http://www.dovepress.com/testimonials.php to read real quotes from published authors. 